\title{
Optimization Problems and Algorithms in Double-layered Food Packing Systems*
}

\author{
Yoshiyuki KARUNO ${ }^{* *}$, Hiroshi NAGAMOCHI ${ }^{* * *}$ and Xiaoming WANG $^{* *}$ \\ ** Department of Mechanical and System Engineering, Kyoto Institute of Technology \\ Goshokaido-cho, Matsugasaki, Sakyo-ku, Kyoto-shi, Kyoto 606-8585, Japan \\ E-mail: karuno@kit.ac.jp \\ *** Department of Applied Mathematics and Physics, Kyoto University \\ Yoshida-honmachi, Sakyo-ku, Kyoto-shi, Kyoto 606-8501, Japan
}

\begin{abstract}
In this paper, we discuss lexicographic bi-criteria combinatorial optimization problems arising in two types of double-layered food packing systems, which we call upright and diagonal types. Such food packing systems are known as so-called automatic combination weighers. The first and second layers in a double-layered food packing system consist of $n$ weighing hoppers and $n$ booster hoppers, respectively. Some amount of food such as a green pepper and a handful of potato chips is thrown into each hopper, and it is called an item. The food packing system performs an operation of choosing a subset $I^{\prime}$ from the set $I$ of the current $2 n$ items to produce a package of the food. Then, the resulting empty hoppers are supplied with next items, and the set $I$ is updated. By repeating the packing operation, a large number of packages are produced one by one. The boosters are just hoppers without weighing function, but the weights of items in the boosters can be known since each type of double-layered food packing systems has its own constructional feature such that they receive next items from the weighing hoppers. The primary objective of lexicographic bi-criteria food packing problems is to minimize the the total weight of chosen items for each package, making the total weight no less than a specified target weight $T$. The second objective is to maximize the total priority of chosen items for each package so that items with longer durations in hoppers are preferably chosen. The priority of an item is given as its duration in hopper. In this paper, we prove that the lexicographic bi-criteria food packing problems can be solved in $O(n T)$ time by dynamic programming if all input data are integral. We also show the executive efficiency of the pseudo-polynomial dynamic programming algorithms by conducting numerical experiments.
\end{abstract}

Key words : Food Packing Equipments, Combinatorial Optimization, Lexicographic Bi-criteria, Target Weight Constraint, Double Layers of Hoppers, Dynamic Programming.

\section{Introduction}

We discuss combinatorial optimization problems arising in automated food packing equipments, so-called automatic combination weighers ${ }^{(1)}$. In this paper, we deal with upright and diagonal types of double-layered food packing systems, which are depicted in Fig. 1 and Fig. 2. The first and second layers in a double-layered food packing system consist of $n$ weighing hoppers and $n$ booster hoppers, respectively. Some amount of food such as a green pepper, a ham, a handful of potato chips, and so on is thrown into each hopper. The food in each hopper can be regarded as an item. Given a set $I$ of current $2 n$ items in hoppers, the system performs an operation of choosing a subset $I^{\prime}(\subseteq I)$ of items to produce a package of the food. Then, the resulting empty hoppers are supplied with next items, and the set $I$ is updated. The system repeats the packing operation to produce a large number of packages one by one.

*Received 30 0ct., 2009 (No. 09-0653) [DOI: 10.1299/jamdsm.4.605]

Copyright (c) 2010 by JSME 
Note that the system always chooses a subset $I^{\prime}$ from the current items without knowing the weights of next new items.

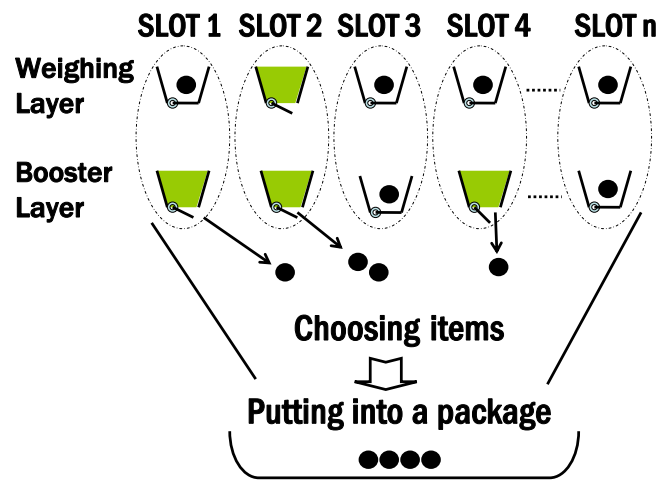

Fig. 1 Upright type of food packing system

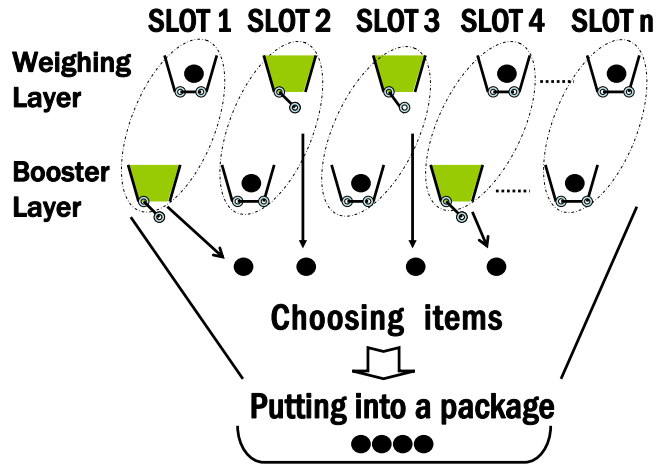

Fig. 2 Diagonal type of food packing system

The boosters are just hoppers without weighing function, but the weights of items in the boosters can be known owing to constructional features of the systems such that the boosters receive next items from the weighing hoppers. An actual example of double-layered food packing system is illustrated by a manufacturer of weighing and packaging equipments ${ }^{(2)}$, which bunches up asparagus shoots.

There also exist some automatic combination weighers which are able to produce about two hundred packages per minute at the maximum ${ }^{(2)}$, i.e., approximately three hundred milliseconds per packing operation. They spend most time in measuring the weights of next new items accurately, and only a few milliseconds may be left for choosing a subset $I^{\prime}$ of items at each packing operation.

For the two types of double-layered food packing systems, we first formulate the problems of choosing a subset $I^{\prime}$ from $I$ at each packing operation as lexicographic bi-criteria combinatorial optimization problems of off-line setting. From a viewpoint of the service conscience, the total weight of chosen items for each package must be no less than a specified target weight $T$. This is referred to as the target weight constraint, which is a hard constraint of automatic combination weighers ${ }^{(1)}$. The primary objective of lexicographic bi-criteria food packing problems is to minimize the total weight of chosen items for each package under the target weight constraint, which aims at attaining the total weight of chosen items for each package as close to the target weight $T$ as possible. In addition, items with longer durations in hoppers would like to be preferably chosen ${ }^{(3)}$. For example, when a system handles some kind of fresh (or raw) food that would like to be vacuum-packed as soon as possible, it is undesirable that items with long durations occur. Following the previous papers ${ }^{(4)-(6)}$, we introduce 
a priority to each current item, and try to maximize the total priority of chosen items for each package as the second objective of lexicographic bi-criteria food packing problems.

Then, we propose $O(n T)$ time algorithms based on dynamic programming to the lexicographic bi-criteria food packing problems for both types of double-layered food packing systems, where all input data are assumed to be integral. We also conduct numerical experiments to examine the executive efficiency of proposed optimizing approaches.

The single-layered food packing systems with weighing hoppers only have been modeled and pseudo-polynomial time algorithms have been proposed $^{(4)-(6)}$. Several numerical results have also been reported ${ }^{(3),(7)-(10)}$.

\section{Problem Description}

We define a slot as a pair of a weighing hopper and the following booster (see again Fig. 1 and Fig. 2). The number of slots is denoted by $n$. We refer to the weighing hopper and following booster in the $i$-th slot as weighing hopper $i$ (or simply hopper $i$ ) and booster $n+i$ (or simply hopper $n+i)$, respectively $(i=1,2, \ldots, n)$. We also refer to the current item in weighing hopper $i$ as item $i$, and refer to the current item in booster $n+i$ as item $n+i$. The total iteration number in a series of packing operations (i.e., the total number of packages to be produced) is denoted by $N$.

\subsection{Constructional Features of Slots}

A weighing hopper sends its current item to the following booster, whenever the booster becomes empty. The booster receives it as the next item. Then, the weighing hopper is supplied with a new item since it becomes empty after sending its item to the following booster. In this paper, we assume that a double-layered food packing system performs each packing operation after all the $2 n$ hoppers become ready (i.e., no empty hopper exists). If a weighing hopper also becomes empty when the following booster becomes empty (such a situation occurs in a double-layered food packing system of upright type), it sends a new item to the following booster after it is supplied with the item.

According to the details of constructional features of slots, we distinguish between the upright and diagonal types of double-layered food packing systems. We classify a doublelayered food packing system into upright type if it cannot choose the current item in a weighing hopper for a package unless it chooses the current item in the following booster. On the other hand, we classify a double-layered system into diagonal type if it can choose the current item in a weighing hopper for a package no matter whether it chooses the current item in the following booster or not. However, the diagonal type cannot simultaneously choose the current two items in any slot for a package (for example, an actual double-layered food packing system has a circular configuration of slots, and the current item in a hopper is send toward the packaging section situated at the center of the circular through the inside of the equipment to be packed when it is chosen. If the aisle of items from a slot to the packaging section is narrow, it may be able to let at most one item pass through). We refer to these restrictions on the choice of a subset of the current $2 n$ items as constructional constraints of slots.

\subsection{Formulations}

Let $\ell$ be the current iteration number of packing operation $(1 \leq \ell \leq N)$, and let $\ell_{i}$ be the iteration number at which current item $i \in\{1,2, \ldots, 2 n\}$ has been brought into a certain empty weighing hopper. Then, we refer to $C_{i}:=\ell-\ell_{i}+1$ as the duration in hopper of the item. In this paper, we define the priority of an item by its duration in hopper, following the previous papers $^{(4)-(6)}$.

The input data below are provided by a problem instance at each packing operation.

- $\quad I=I_{\mathrm{W}} \cup I_{\mathrm{b}}$ : set of the current $2 n$ items, where $I_{\mathrm{W}}=\{i \mid i=1,2, \ldots, n\}$ is a set of $n$ items in weighing hoppers, and $I_{\mathrm{b}}=\{n+i \mid i=1,2, \ldots, n\}$ is a set of $n$ items in boosters. 
- $w_{i}\left(\leq w_{\max }\right)$ : positive integer weight of item $i \in I_{\mathrm{W}}$, which is assumed to be bounded from above by a given positive integer $w_{\max }$.

- $\quad b_{i}\left(\leq w_{\max }\right)$ : positive integer weight of item $n+i \in I_{\mathrm{b}}$, which is also bounded from above by the $w_{\max }$ since booster $n+i$ has received the item from weighing hopper $i$.

- $\quad \gamma_{i}\left(:=C_{i}\right)$ : priority of item $i \in I_{\mathrm{W}}$, which is assumed to be a positive integer.

- $\quad \lambda_{i}\left(:=C_{n+i}\right)$ : priority of item $n+i \in I_{\mathrm{b}}$, which is also assumed to be a positive integer.

- $T$ : target weight of a single package, which is assumed to be a positive integer.

We can compute the following values from the above input data.

- $\quad W=\sum_{i=1}^{n} w_{i}$ : total weight of $n$ items in all weighing hoppers.

- $\quad B=\sum_{i=1}^{n} b_{i}$ : total weight of $n$ items in all boosters.

- $H=\sum_{i=1}^{n} \max \left\{w_{i}, b_{i}\right\}$ : total weight of heavier items in $n$ slots.

A solution of lexicographic bi-criteria food packing problems at each packing operation can be represented by $0-1$ vectors $x=\left(x_{1}, x_{2}, \ldots, x_{n}\right)$ and $y=\left(y_{1}, y_{2}, \ldots, y_{n}\right)$, where

$x_{i}= \begin{cases}1 & \text { if item } i \in I_{\mathrm{W}} \text { is chosen, } \\ 0 & \text { otherwise }\end{cases}$
$y_{i}= \begin{cases}1 & \text { if item } n+i \in I_{\mathrm{b}} \text { is chosen, } \\ 0 & \text { otherwise }\end{cases}$

By using the vectors $x$ and $y$, the food packing problems are formulated as follows.

LEXICO_PACK_U (for Upright Type)

$$
\begin{array}{ll}
\operatorname{minimize} & f_{1}(x, y)=\sum_{i=1}^{n} w_{i} x_{i}+\sum_{i=1}^{n} b_{i} y_{i}, \\
\text { maximize } & f_{2}(x, y)=\sum_{i=1}^{n} \gamma_{i} x_{i}+\sum_{i=1}^{n} \lambda_{i} y_{i}, \\
\text { subject to } & \sum_{i=1}^{n} w_{i} x_{i}+\sum_{i=1}^{n} b_{i} y_{i} \geq T, \\
x_{i}-y_{i} \leq 0, \quad i=1,2, \ldots, n, \\
x_{i}, y_{i} \in\{0,1\}, \quad i=1,2, \ldots, n .
\end{array}
$$

LEXICO_PACK_D (for Diagonal Type)

$$
\begin{array}{ll}
\operatorname{minimize} & f_{1}(x, y)=\sum_{i=1}^{n} w_{i} x_{i}+\sum_{i=1}^{n} b_{i} y_{i}, \\
\text { maximize } & f_{2}(x, y)=\sum_{i=1}^{n} \gamma_{i} x_{i}+\sum_{i=1}^{n} \lambda_{i} y_{i}, \\
\text { subject to } & \sum_{i=1}^{n} w_{i} x_{i}+\sum_{i=1}^{n} b_{i} y_{i} \geq T, \\
x_{i}+y_{i} \leq 1, \quad i=1,2, \ldots, n, \\
x_{i}, y_{i} \in\{0,1\}, \quad i=1,2, \ldots, n .
\end{array}
$$

In LEXICO_PACK_U, the first objective $f_{1}$ of Eq. (3) aims at attaining the total weight of chosen items as close to the target weight $T$ as possible, together with Eq. (5) (i.e., the target weight constraint). The second objective $f_{2}$ of Eq. (4) is introduced to expect that items with longer durations in hoppers are preferably chosen. The upright type cannot choose the current item $i \in I_{\mathrm{W}}$ unless it chooses the item $n+i \in I_{\mathrm{b}}$. Thus, if the $y_{i}$ is set to be zero, the $x_{i}$ must also be zero. That is, Eq. (6) represents the constructional constraint of slots. Equation (7) expresses the binary constraint of variables. Note that there are three possible cases for a 
couple of $x_{i}$ and $y_{i}$, i.e., $\left\langle x_{i}, y_{i}\right\rangle \in\{\langle 0,0\rangle,\langle 0,1\rangle,\langle 1,1\rangle\}$. A solution $(x, y)$ satisfying Eqs. (5)-(7) is referred to as a feasible solution of LEXICO_PACK_U.

In LEXICO_PACK_D, Eq. (9) replaces Eq. (6) as the constructional constraint of slots. The diagonal type cannot simultaneously choose the current two items in any slot. Hence, at least either $x_{i}$ or $y_{i}$ must be zero for any $i=1,2, \ldots, n$. Thus, also in LEXICO_PACK D, there are three possible cases for a couple of $x_{i}$ and $y_{i}$, i.e., $\left\langle x_{i}, y_{i}\right\rangle \in\{\langle 0,0\rangle,\langle 0,1\rangle,\langle 1,0\rangle\}$. A solution $(x, y)$ satisfying Eqs. (8)-(10) is referred to as a feasible solution of LEXICO_PACK_D.

By introducing a bound $Q \in\{W+B, H\}$, we here assume that

$$
w_{\max }<T \leq Q \quad\left(=O\left(n w_{\max }\right)\right) .
$$

Precisely speaking, we assume that $w_{\max }<T \leq W+B\left(=\sum_{i=1}^{n}\left(w_{i}+b_{i}\right)\right)$ for upright type, and that $w_{\max }<T \leq H\left(=\sum_{i=1}^{n} \max \left\{w_{i}, b_{i}\right\}\right)$ for diagonal type. Since $\Sigma_{i=1}^{n}\left(w_{i}+b_{i}\right)=$ $O\left(n w_{\max }\right)$ and $\sum_{i=1}^{n} \max \left\{w_{i}, b_{i}\right\}=O\left(n w_{\max }\right)$, we treat the problems LEXICO_PACK_U and LEXICO_PACK_D on the above common assumption for the sake of convenience.

Moreover, for both food packing problems, we denote by $T^{*}$ the minimum of the total weight of a feasible solution $(x, y)$, and by $(x, y)=(\hat{x}, \hat{y})$ such a feasible solution that attains the total weight with $T^{*}\left(=f_{1}(\hat{x}, \hat{y})\right)$. An optimal solution $(x, y)=\left(x^{*}, y^{*}\right)$ is defined as a feasible solution such that it satisfies $f_{1}\left(x^{*}, y^{*}\right)=T^{*}$ and $f_{2}\left(x^{*}, y^{*}\right) \geq f_{2}(\hat{x}, \hat{y})$ for any $(\hat{x}, \hat{y})$. The value $f_{2}\left(x^{*}, y^{*}\right)$ of the second objective is the maximum of the total priority of a feasible solution under the condition that the primary objective is optimized. We denote by $G^{*}=f_{2}\left(x^{*}, y^{*}\right)$ the conditionally maximum total priority. The problems ask to find an optimal solution $\left(x^{*}, y^{*}\right)$.

When we are asked to find a feasible solution $(x, y)=(\hat{x}, \hat{y})$, we call the problems PRIMAL_PACK_U and PRIMAL_PACK_D respectively, which ignore the total priority of chosen items for a package.

\section{Dynamic Programming}

We define the following $0-1$ state variables $u_{k}(p)(k=1,2, \ldots, n, p=0,1, \ldots, Q)$.

$$
\begin{aligned}
& u_{k}(p)=1 \Longleftrightarrow\left\{\begin{array}{l}
\text { There exists a pair of (partial) } 0 \text { - } 1 \text { vectors }\left(x_{1}, x_{2}, \ldots, x_{k}\right) \text { and } \\
\left(y_{1}, y_{2}, \ldots, y_{k}\right) \text { such that it satisfies } \sum_{i=1}^{k} w_{i} x_{i}+\sum_{i=1}^{k} b_{i} y_{i}=p, \\
\text { and satisfies either } x_{i}-y_{i} \leq 0 \text { for LEXICO_PACK_U or } \\
x_{i}+y_{i} \leq 1 \text { for LEXICO_PACK_D. }
\end{array}\right. \\
& u_{k}(p)=0 \Longleftrightarrow \text { There exists no such a pair of (partial) } 0 \text {-1 vectors. }
\end{aligned}
$$

We also define variables $z_{k}(p)(k=1,2, \ldots, n, p=0,1, \ldots, Q)$ to record the total priority $\sum_{i=1}^{k} \gamma_{i} x_{i}+\sum_{i=1}^{k} \lambda_{i} y_{i}$ of the (partial) $0-1$ vectors $\left(x_{1}, x_{2}, \ldots, x_{k}\right)$ and $\left(y_{1}, y_{2}, \ldots, y_{k}\right)$ such that it satisfies $\sum_{i=1}^{k} w_{i} x_{i}+\sum_{i=1}^{k} b_{i} y_{i}=p$ (and satisfies either $x_{i}-y_{i} \leq 0$ for LEXICO_PACK_U or $x_{i}+y_{i} \leq 1$ for LEXICO_PACK_D). Moreover, we introduce three kinds of additional variables $q_{k}^{(1)}(p), q_{k}^{(2)}(p)$ and $q_{k}^{(3)}(p)(k=1,2, \ldots, n, p=0,1, \ldots, Q)$ in order to maintain the maximum of the total priority for a possible case in the $z_{k}(p)$. That is, these variables correspond to the three possible cases for a couple of $x_{k}$ and $y_{k}$ (recall the feasibility of a solution mentioned in the previous section).

The recursives of dynamic programming for LEXICO_PACK_U are presented as follows. For $p=0,1, \ldots, W+B$,

$$
\begin{aligned}
& u_{1}(p)= \begin{cases}1 & \text { if } p \in\left\{0, b_{1}, w_{1}+b_{1}\right\} \\
0 & \text { otherwise },\end{cases} \\
& z_{1}(p)= \begin{cases}0 & \text { if } p=0, \\
\lambda_{1} & \text { if } p=b_{1} \\
\gamma_{1}+\lambda_{1} & \text { if } p=w_{1}+b_{1} \\
-1 & \text { otherwise }\end{cases}
\end{aligned}
$$


and for $k=2,3, \ldots, n, p=0,1, \ldots, W+B$,

$$
\begin{aligned}
& u_{k}(p)= \begin{cases}1 \quad \text { if } u_{k-1}(p)=1, \text { or } & \text { if }\left(p-b_{k} \geq 0 \text { and } u_{k-1}\left(p-b_{k}\right)=1\right), \text { or } \\
\text { if }\left(p-w_{k}-b_{k} \geq 0 \text { and } u_{k-1}\left(p-w_{k}-b_{k}\right)=1\right), & \text { otherwise, }\end{cases} \\
& q_{k}^{(1)}(p)= \begin{cases}z_{k-1}(p) & \text { if } u_{k-1}(p)=1, \\
-1 & \text { otherwise, }\end{cases} \\
& q_{k}^{(2)}(p)= \begin{cases}z_{k-1}\left(p-b_{k}\right)+\lambda_{k} & \text { if }\left(p-b_{k} \geq 0 \text { and } u_{k-1}\left(p-b_{k}\right)=1\right), \\
-1 & \text { otherwise, }\end{cases} \\
& q_{k}^{(3)}(p)= \begin{cases}z_{k-1}\left(p-w_{k}-b_{k}\right)+\gamma_{k}+\lambda_{k} \\
-1 & \text { if }\left(p-w_{k}-b_{k} \geq 0 \text { and } u_{k-1}\left(p-w_{k}-b_{k}\right)=1\right), \\
z_{k}(p)= & \max \left\{q_{k}^{(1)}(p), q_{k}^{(2)}(p), q_{k}^{(3)}(p)\right\} .\end{cases}
\end{aligned}
$$

We call the dynamic programming Lexico_DP(U). The correctness is explained as follows. In LEXICO_PACK_U, because of the constructional constraint of slots (see Eq. (6)), there are three possible cases for a couple of $x_{k}$ and $y_{k}$, i.e., $\left\langle x_{k}, y_{k}\right\rangle \in\{\langle 0,0\rangle,\langle 0,1\rangle,\langle 1,1\rangle\}$. If $u_{k}(p)=1$ holds due to $u_{k-1}(p)=1$, the first case $\left\langle x_{k}, y_{k}\right\rangle=\langle 0,0\rangle$ (i.e., neither item $k$ nor item $n+k$ is chosen) is a candidate setting in an optimal solution, and hence the $q_{k}^{(1)}(p)$ records the total priority $z_{k-1}(p)$. If $u_{k}(p)=1$ holds due to $p-b_{k} \geq 0$ and $u_{k-1}\left(p-b_{k}\right)=1$, the second case $\left\langle x_{k}, y_{k}\right\rangle=\langle 0,1\rangle$ (i.e., item $k$ is not chosen, while item $n+k$ is chosen) is a candidate setting in an optimal solution, and hence the $q_{k}^{(2)}(p)$ records the total priority $z_{k-1}\left(p-b_{k}\right)+\lambda_{k}$. If $u_{k}(p)=1$ holds due to $p-w_{k}-b_{k} \geq 0$ and $u_{k-1}\left(p-w_{k}-b_{k}\right)=1$, the third case $\left\langle x_{k}, y_{k}\right\rangle=\langle 1,1\rangle$ (i.e., both items $k$ and $n+k$ are chosen) is a candidate setting in an optimal solution, and hence the $q_{k}^{(3)}(p)$ records the total priority $z_{k-1}\left(p-w_{k}-b_{k}\right)+\gamma_{k}+\lambda_{k}$. Let $z_{k}(p)=\max \left\{q_{k}^{(1)}(p), q_{k}^{(2)}(p), q_{k}^{(3)}(p)\right\}$ (which breaks ties arbitrarily). Then, the corresponding pair of (partial) $0-1$ vectors $\left(x_{1}, x_{2}, \ldots, x_{k}\right)$ and $\left(y_{1}, y_{2}, \ldots, y_{k}\right)$ to the $z_{k}(p)$ has the maximum of the total priority among pairs of (partial) $0-1$ vectors such that they satisfy $\sum_{i=1}^{k} w_{i} x_{i}+\sum_{i=1}^{k} b_{i} y_{i}=p$.

The initializations of Eqs. (12) and (13) are trivial by definition, and Eqs. (14)-(18) are derived from the above discussion.

The computation of all $u_{k}(p), z_{k}(p), q_{k}^{(1)}(p), q_{k}^{(2)}(p)$ and $q_{k}^{(3)}(p)$ requires $O(n(W+B))=$ $O\left(n^{2} w_{\max }\right)$ time (see Eq. (11)). After computing all $u_{k}(p)$ and all $z_{k}(p)$, we find a minimum $p=p_{\min }$ such that it satisfies $u_{n}(p)=1$ and $p \geq T$, which requires additionally $O(W+B)=O\left(n w_{\max }\right)$ time (see again Eq. (11)). We can see that $p_{\min }=T^{*}$ (i.e., the minimum total weight) and $z_{n}\left(p_{\min }\right)=G^{*}$ (i.e., the conditionally maximum total priority). Thus, Lexico_DP(U) can compute the $T^{*}$ and $G^{*}$ of LEXICO_PACK_U in $O\left(n^{2} w_{\max }\right)$ time. By backtracking the computation process, we can also construct an optimal solution $(x, y)=\left(x^{*}, y^{*}\right)$.

Lemma 1 For an instance of LEXICO_PACK_U, algorithm Lexico_DP(U) can obtain an optimal solution $(x, y)=\left(x^{*}, y^{*}\right)$ in $O\left(n^{2} w_{\max }\right)$ time.

The recursives of dynamic programming for LEXICO_ PACK_D are presented as follows. For $p=0,1, \ldots, H$,

$$
u_{1}(p)= \begin{cases}1 & \text { if } p \in\left\{0, b_{1}, w_{1}\right\} \\ 0 & \text { otherwise }\end{cases}
$$




$$
z_{1}(p)= \begin{cases}0 & \text { if } p=0, \\ \lambda_{1} & \text { if } p=b_{1}\left(\neq w_{1}\right), \\ \gamma_{1} & \text { if } p=w_{1}\left(\neq b_{1}\right), \\ \max \left\{\gamma_{1}, \lambda_{1}\right\} & \text { if } p=w_{1}=b_{1}, \\ -1 & \text { otherwise, }\end{cases}
$$

and for $k=2,3, \ldots, n, p=0,1, \ldots, H$,

$$
\begin{aligned}
& u_{k}(p)=\left\{\begin{array}{cc}
1 \quad & \text { if } u_{k-1}(p)=1, \text { or } \\
& \text { if }\left(p-b_{k} \geq 0 \text { and } u_{k-1}\left(p-b_{k}\right)=1\right), \text { or } \\
& \text { if }\left(p-w_{k} \geq 0 \text { and } u_{k-1}\left(p-w_{k}\right)=1\right), \\
0 \quad \text { otherwise }
\end{array}\right. \\
& q_{k}^{(1)}(p)= \begin{cases}z_{k-1}(p) & \text { if } u_{k-1}(p)=1, \\
-1 & \text { otherwise, }\end{cases} \\
& q_{k}^{(2)}(p)= \begin{cases}z_{k-1}\left(p-b_{k}\right)+\lambda_{k} & \text { if }\left(p-b_{k} \geq 0 \text { and } u_{k-1}\left(p-b_{k}\right)=1\right), \\
-1 & \text { otherwise, }\end{cases} \\
& q_{k}^{(3)}(p)= \begin{cases}z_{k-1}\left(p-w_{k}\right)+\gamma_{k} & \text { if }\left(p-w_{k} \geq 0 \text { and } u_{k-1}\left(p-w_{k}\right)=1\right), \\
-1 & \text { otherwise, }\end{cases} \\
& z_{k}(p)=\max \left\{q_{k}^{(1)}(p), q_{k}^{(2)}(p), q_{k}^{(3)}(p)\right\} \text {. }
\end{aligned}
$$

We call the dynamic programming Lexico_DP(D). Also in LEXICO_PACK_D, because of the constructional constraint of slots (see Eq. (9)), there are three possible cases for a couple of $x_{k}$ and $y_{k}$, i.e., $\left\langle x_{k}, y_{k}\right\rangle \in\{\langle 0,0\rangle,\langle 0,1\rangle,\langle 1,0\rangle\}$. Notice that the last case $\langle 1,0\rangle$ in LEXICO_PACK_D replaces $\langle 1,1\rangle$ in LEXICO_PACK_U. If $u_{k}(p)=1$ holds due to $p-w_{k} \geq 0$ and $u_{k-1}\left(p-w_{k}\right)=1$, the replaced last case $\left\langle x_{k}, y_{k}\right\rangle=\langle 1,0\rangle$ (i.e., item $k$ is chosen, while item $n+k$ is not chosen) is a candidate setting in an optimal solution. Hence, in Lexico_DP(D), the $q_{k}^{(3)}(p)$ records the total priority $z_{k-1}\left(p-w_{k}\right)+\gamma_{k}$. The discussion on its correctness is similar to that for Lexico_DP(U). Also, it is obvious that Lexico_DP(D) has the same time complexity as that of Lexico_DP(U).

Lemma 2 For an instance of LEXICO_PACK_D, algorithm Lexico_DP(D) can obtain an optimal solution $(x, y)=\left(x^{*}, y^{*}\right)$ in $O\left(n^{2} w_{\max }\right)$ time.

In order to improve the time complexities of Lexico_ DP(U) and Lexico_DP(D), we provide the following property of optimal solutions $(x, y)=\left(x^{*}, y^{*}\right)$.

Lemma 3 For an instance of LEXICO_PACK_U and LEXICO_PACK_D, the total weight $T^{*}\left(=f_{1}\left(x^{*}, y^{*}\right)\right)$ of an optimal solution $\left(x^{*}, y^{*}\right)$ satisfies the following inequalities.

$$
T \leq T^{*}<T+w_{\max }
$$

Proof. Let $\left(x^{*}, y^{*}\right)=\left(\left(x_{1}^{*}, x_{2}^{*}, \ldots, x_{n}^{*}\right),\left(y_{1}^{*}, y_{2}^{*}, \ldots, y_{n}^{*}\right)\right)$ be an optimal solution. Since the optimal solution $\left(x^{*}, y^{*}\right)$ satisfies the target weight constraint (see Eq. (5)), it is clear that the former of Eq. (23) holds.

For LEXICO_PACK_U, by the optimality of $\left(x^{*}, y^{*}\right)$, it holds $f_{1}\left(x^{*}, y^{*}\right)-b_{k}<T$ for any item $n+k$ with $\left\langle x_{k}^{*}, y_{k}^{*}\right\rangle=\langle 0,1\rangle$, and it also holds $f_{1}\left(x^{*}, y^{*}\right)-w_{k}<T$ for any item $k$ with $\left\langle x_{k}^{*}, y_{k}^{*}\right\rangle=\langle 1,1\rangle$. There may be an item $n+k$ with $\left\langle x_{k}^{*}, y_{k}^{*}\right\rangle=\langle 1,1\rangle$ such that it satisfies $f_{1}\left(x^{*}, y^{*}\right)-b_{k} \geq T$. However, deleting such an item from the optimal solution, it is not a feasible solution any longer since it cannot meet the constructional constraint of slots. Therefore, the latter of the inequalities holds for LEXICO_PACK_U.

For LEXICO_PACK_D, by the optimality of $\left(x^{*}, y^{*}\right)$, it holds $f_{1}\left(x^{*}, y^{*}\right)-w_{k}<T$ for any item $k$ with $\left\langle x_{k}^{*}, y_{k}^{*}\right\rangle=\langle 1,0\rangle$, and it also holds $f_{1}\left(x^{*}, y^{*}\right)-b_{k}<T$ for any item $n+k$ with $\left\langle x_{k}^{*}, y_{k}^{*}\right\rangle=\langle 0,1\rangle$, which implies the latter of Eq. (23). 
By Lemma 3, the computation interval $[0, Q]$ of parameter $p$ for $u_{k}(p), z_{k}(p), q_{k}^{(1)}(p)$, $q_{k}^{(2)}(p)$ and $q_{k}^{(3)}(p)$ in Lexico_DP(U) and Lexico_DP(D) can be reduced to $\left[0, \min \left\{T+w_{\max }-\right.\right.$ $1, Q\}]$. We call the improved (faster) algorithms Lexico_FDP(U) and Lexico_FDP(D), respectively. Together with assumption of Eq. (11), we obtain the following theorem.

Theorem 1 For an instance of LEXICO_PACK_U (resp., LEXICO_PACK_D), algorithm Lexico_FDP(U) (resp., Lexico_FDP(D)) can obtain an optimal solution $(x, y)=\left(x^{*}, y^{*}\right)$ in $O(n T)$ time.

The improved time complexities of dynamic programming algorithms of Lexico_FDP(U) and Lexico_ FDP(D) are also pseudo-polynomial, but are linear in $n$. By applying Theorem 1, PRIMAL_PACK_U and PRIMAL_PACK_D can also be solved in $O(n T)$ time. We refer to the corresponding algorithms as Primal_FDP(U) and Primal_FDP(D) respectively, which ignore the total priority of chosen items.

\section{Numerical Experiments}

In this section, by means of numerical experiments, we examine the executive efficiency of proposed optimizing approaches. We also report the results of Primal_FDP(U) and Primal_FDP(D), which ignore the durations in hoppers of items. As an implementation of these algorithms, we employ Lexico_FDP(U) and Lexico_FDP(D), but give a constant priority to each item, which is independent of the duration of the item. The program is written in C. It is compiled by Microsoft Visual $\mathrm{C}++$, and is run on a personal computer with Intel Pentium $\mathrm{M}$ CPU (1.20 GHz) and 1GB memory (Panasonic CF-W4HWSAXC). Problem instances to be tested are randomly generated as follows.

- The number of slots: $n \in\{5,10,15,20\}$ (for example, an actual double-layered food packing system with seven slots, i.e., $n=7$, is illustrated by a manufacturer of weighing and packaging equipments ${ }^{(2)}$, which makes thirty bundles of asparagus shoots per minute).

- Integer weights: $w_{i}$ 's (and hence $b_{i}$ 's) are uniformly random integers in each of intervals $[35,55]$ and $[175,275]$.

- Target weight: $T \in\{180,900\}$.

- The total iteration number of packing operations: $N=10000$.

In Tables 1-3, each of the data indicates the mean value for ten series of $N$ packing operations (i.e., $10000 \times 10$ packages are produced to obtain each of the data). The notations used in the tables have the following meanings.

- $\quad W_{\text {mean: }}$ the mean value of total weights for $N$ packages.

- $\quad R_{\mathrm{T}}$ : the accomplished rate of packages with total weight equal to the target weight $T$ during a series of $N$ packing operations, i.e.,

$$
R_{\mathrm{T}}=\frac{\text { The number of packages with total weight equal to } T}{\text { The total iteration number of packing operations, } N} \times 100[\%] \text {. }
$$

- $\quad C_{\max }, C_{\text {mean: }}$ the maximum and mean durations over all items brought in the doublelayered food packing systems during a series of $N$ packing operations.

- CPU: the execution time of CPU required to obtain an optimal solution at each packing operation.

In Table 1 , the deviations of the mean values $W_{\text {mean }}$ of total weights from the target weight $T$ are less than 1 [\%] of the $T$ for all algorithms. Also the algorithms obtain the accomplished rates $R_{\mathrm{T}}$ greater than 95 [\%] for instances with $n \geq 10$.

In Table 2, the $C_{\max }$ by Primal_FDP(U) is greater than 100 for instances with $n \geq 10$, while Lexico_FDP(U) attains the $C_{\max }$ less than 40 for any tested instance with up to $n=20$. The $C_{\max }$ by Primal_FDP(D) is greater than 300 for instances with $n \geq 10$, while the $C_{\max }$ 
Table 1 Effect upon the total weight

\begin{tabular}{cccc}
\hline \multicolumn{4}{c}{ Upright } \\
\hline $\begin{array}{c}\text { The number } \\
\text { of slots, } n\end{array}$ & $W_{\text {mean }}$ & $R_{\mathrm{T}}[\%]$ \\
\hline 5 & 181.4 & 63.6 \\
10 & 180.1 & 95.9 \\
15 & 180.0 & 98.6 \\
20 & 180.0 & 99.4 \\
\hline \multicolumn{4}{c}{ Lexico_FDP(U) } \\
\hline 5 & 181.4 & 63.2 \\
10 & 180.1 & 96.4 \\
15 & 180.0 & 99.3 \\
20 & 180.0 & 99.9 \\
\hline \multicolumn{5}{c}{$w_{i}, b_{i} \in[35,55], T=180$} \\
\hline \multicolumn{5}{c}{ Primal_FDP(D) } \\
\hline The number & $R_{\mathrm{T}}[\%]$ \\
\hline of slots, $n$ & Diagonal & 76.5 \\
\hline 5 & $W_{\text {mean }}$ & 98.2 \\
10 & 181.2 & 99.3 \\
15 & 180.1 & 99.6 \\
20 & 180.0 & 76.7 \\
\hline \multicolumn{5}{c}{ Lexico_FDP(D) } \\
\hline 5 & 180.0 & 99.1 \\
10 & 181.2 & 99.9 \\
15 & 180.0 & 99.9 \\
20 & 180.0 & $w_{i}, b_{i} \in[35,55], T=180$ \\
\hline \multicolumn{3}{c}{}
\end{tabular}

by Lexico_FDP(D) is less than 30. The proposed optimizing approaches perform well for reducing the maximum duration $C_{\max }$.

When we apply the single criterion approaches Primal_FDP(U) and Primal_FDP(D), the performance of upright type seems to be far better than that of diagonal type for the maximum duration $C_{\max }$. However, the bi-criteria approach Lexico_FDP(D) recovers the performance of diagonal type.

In Table 3, improved algorithms Lexico_FDP(U) and Lexico_FDP(D) actually run faster than the basic ones Lexico_DP(U) and Lexico_DP(D) with common $Q=W+B(\approx 2 H)$, respectively.

\section{Conclusions}

In this paper, we dealt with double-layered food packing systems. Due to the constructional constraints of slots, we had to distinguish between upright and diagonal types. We newly formulated food packing problems at each packing operation as lexicographic bi-criteria combinatorial optimization problems, LEXICO_PACK_U and LEXICO_PACK_D. Then, we proposed $O(n T)$ time algorithms based on dynamic programming to the problems, where $n$ is the number of slots and $T$ is the target weight of a single package. By numerical experiments, we observed that the proposed approaches have a significant effect for reducing the maximum duration in hoppers. We also observed that the $O(n T)$ time algorithms actually run faster than the basic recursives with $O\left(n^{2} w_{\max }\right)$ time complexity, where $w_{\max }$ is the upper limit of weights of items. The improved algorithms can be used as tools of simulation studies, and they have the potential for practical use without becoming a bottleneck part of the actual food packing equipments.

\section{Acknowledgment}

We were allowed to give the presentation of a part of this paper at the Fourth International Symposium on Scheduling (ISS 2009), July 4-6, 2009, Nagoya, Aichi, Japan. We would like to express our gratitude to the organizing, executive and international program committees of 
Table 2 Effect upon the maximum and mean durations

\begin{tabular}{|c|c|c|}
\hline \multicolumn{3}{|c|}{ Upright } \\
\hline The number & \multicolumn{2}{|c|}{ Primal_FDP(U) } \\
\hline of slots, $n$ & $C_{\max }$ & $C_{\text {mean }}$ \\
\hline 5 & 27 & 2.5 \\
\hline 10 & 113 & 5.0 \\
\hline 15 & 290 & 7.5 \\
\hline \multirow[t]{2}{*}{20} & 531 & 9.9 \\
\hline & \multicolumn{2}{|c|}{ Lexico_FDP(U) } \\
\hline 5 & 22 & 2.5 \\
\hline 10 & 33 & 5.0 \\
\hline 15 & 34 & 7.4 \\
\hline \multirow[t]{2}{*}{20} & 38 & 9.9 \\
\hline & \multicolumn{2}{|c|}{$w_{i}, b_{i} \in[35,55], T=180$} \\
\hline \multicolumn{3}{|c|}{ Diagonal } \\
\hline The number & \multicolumn{2}{|c|}{ Primal_FDP(D) } \\
\hline of slots, $n$ & $C_{\max }$ & $C_{\text {mean }}$ \\
\hline 5 & 42 & 2.5 \\
\hline 10 & 359 & 4.9 \\
\hline 15 & 989 & 7.4 \\
\hline \multirow[t]{2}{*}{20} & 1775 & 9.9 \\
\hline & \multicolumn{2}{|c|}{ Lexico_FDP(D) } \\
\hline 5 & 25 & 2.5 \\
\hline 10 & 28 & 4.9 \\
\hline 15 & 27 & 7.4 \\
\hline 20 & 28 & 9.9 \\
\hline & & $T=180$ \\
\hline
\end{tabular}

Table 3 Execution times of algorithms

\begin{tabular}{|c|c|c|}
\hline \multicolumn{3}{|c|}{ Upright } \\
\hline The number & \multicolumn{2}{|c|}{ CPU time $[\mathrm{msec}]$} \\
\hline of slots, $n$ & Lexico_DP(U) & Lexico_FDP(U) \\
\hline 5 & 0.2 & 0.1 \\
\hline 10 & 1.2 & 0.3 \\
\hline 15 & 2.8 & 0.5 \\
\hline 20 & 5.0 & 0.7 \\
\hline \multicolumn{3}{|c|}{$w_{i}, b_{i} \in[35,55], T=180$} \\
\hline 5 & 1.0 & 0.5 \\
\hline 10 & 5.5 & 1.3 \\
\hline 15 & 13.6 & 2.2 \\
\hline 20 & 25.3 & 3.1 \\
\hline \multicolumn{3}{|c|}{$w_{i}, b_{i} \in[175,275], T=900$} \\
\hline \multicolumn{3}{|c|}{ Diagonal } \\
\hline The number & \multicolumn{2}{|c|}{ CPU time $[\mathrm{msec}]$} \\
\hline of slots, $n$ & Lexico_DP(D) & Lexico_FDP(D) \\
\hline 5 & 0.2 & 0.1 \\
\hline 10 & 1.1 & 0.4 \\
\hline 15 & 2.4 & 0.6 \\
\hline 20 & 4.3 & 0.8 \\
\hline \multicolumn{3}{|c|}{$w_{i}, b_{i} \in[35,55], T=180$} \\
\hline 5 & 1.0 & 0.6 \\
\hline 10 & 5.0 & 1.5 \\
\hline 15 & 12.3 & 2.5 \\
\hline 20 & 22.2 & 3.4 \\
\hline
\end{tabular}

the symposium. Also we would like to thank two anonymous referees for their suggestive comments to the first version of this paper.

\section{References}

( 1 ) Morinaka, H., Automatic Combination Weigher for Product Foods (in Japanese), Journal of the Japan Society of Mechanical Engineers, Vol. 103, (2000), pp. 130-131. 
( 2 ) ISHIDA CO., LTD., http: //www . ishida.co.jp (Products, Weighing and Packaging, in Japanese), (2009), [accessed January 2009].

( 3 ) Kameoka, K. and Nakatani, M., Feed Control Criterion for a Combination Weigher and its Effects (in Japanese), Transactions of the Society of Instrument and Control Engineers, Vol. 37, (2001), pp. 911-915.

( 4 ) Imahori, S., Karuno, Y., Nagamochi, H., and Wang, X., Efficient Algorithms for Combinatorial Food Packing Problems, Proceedings of the 11th International Conference on Humans and Computers (HC 2008), November 20-23, 2008, Nagaoka, Niigata, Japan, (2008), pp. 317-322.

( 5 ) Karuno, Y., Nagamochi, H., and Ohshima, Y., A Dynamic Programming Approach for a Food Packing Problem (in Japanese), Transactions of the Japan Society of Mechanical Engineers, Series C, Vol. 72, (2006), pp. 1390-1397.

( 6 ) Karuno, Y., Nagamochi, H., and Wang, X., Bi-criteria Food Packing by Dynamic Programming, Journal of the Operations Research Society of Japan, Vol. 50, (2007), pp. 376-389.

( 7 ) Kameoka, K., Nakatani, M., and Inui, N., Phenomena in Probability and Statistics Found in a Combinatorial Weigher (in Japanese), Transactions of the Society of Instrument and Control Engineers, Vol. 36, (2000), pp. 388-394.

( 8 ) Murakami, Y., Kurata, J., Uchiyama, H. Taniya, K., and Kawai, M., Efficient Algorithm for Solving a Bag-packing Problem by Excluding Search Space (in Japanese), Transactions of the Japan Society of Mechanical Engineers, Series C, Vol. 69, (2003), pp. 3431-3438.

( 9 ) Murakami, Y., Kurata, J., Uchiyama, H., and Ueno, T., Characterization of Infeasible Solutions in a Bag-packing Problem for Achieving Desired Weight (in Japanese), Transactions of the Society of Instrument and Control Engineers, Vol. 38, (2002), pp. 784-791.

(10) Murakami, Y., Uchiyama, H., Kurata, J., and Kotera, T., High Efficiency of Weighing Machine for Achieving Desired Weights by Tuning Input Amount (in Japanese), Transactions of the Japan Society of Mechanical Engineers, Series C, Vol. 74, (2008), pp. 1920-1925. 